\title{
Pathophysiology of the Unexplained Stillbirth
}

\author{
Anna Maria Lavezzi, ${ }^{1, *}$ and Francesco Piscioli ${ }^{2}$

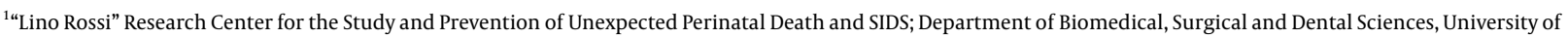 \\ Milan \\ ${ }^{2}$ Institute of Pathology, Hospital of Rovereto (Trento), Italy \\ "Corresponding author: Anna Maria Lavezzi, "Lino Rossi” Research center for the study and prevention of unexpected perinatal death and SIDS - Department of Biomedical, \\ Surgical and Dental Sciences, University of Milan, Via della Commenda 19, 20122 Milan, Italy. Tel: +39-0250320821, E-mail: anna.lavezzi@unimi.it \\ Received 2017 June 21; Accepted 2017 July 20.
}

Although with the introduction of better management of high-risk pregnancies, the stillbirth rate has been notably reduced during the last decades, intrauterine fetal death remains a major health problem, accounting for a large part of perinatal mortality in developed countries (1).

Stillbirth represents a very emotional distressing event for both parents and obstetricians. In recent years the universal recommendation to submit the fetal losses to careful autopsy, including placenta and umbilical cord examination, has in many cases allowed to highlight the cause of death, generally falling into one of three following categories: 1) fetal pathologies (malformations, chromosomal abnormalities, genetic mutations, etc.); 2) adnexa pathologies (placental abruption, umbilical cord accidents, chorioamnionitis, etc.); 3) maternal pathologies (infections, uncontrolled diabetes, high blood pressure, advanced age, obesity, etc). However, despite extensive clinical and anatomopathological examinations, a specific cause of death continues to be unidentified in almost half of cases. These deaths are classified as "unexplained stillbirths" (2).

In Italy, the "Lino Rossi" Research Center of Milan University, as national referral center for the application of the Italian law n.31/2006 "Regulations for Diagnostic Post Mortem Investigation in Victims of Sudden Infant Death Syndrome (SIDS) and Unexpected Fetal Death", in more than 10 years has collected and thoroughly examined a wide set of unexplained sudden fetal deaths $(3,4)$.

This law decrees in fact, regarding the fetuses who died without any apparent cause, that they must be submitted to a thorough diagnostic post-mortem investigation that includes, above all, the examination of the autonomic nervous system. The anatomopathological protocol developed by the Lino Rossi Research Center for this specific purpose, concerns above all the analysis of the main centers that control the vital functions, prevalently located in the brainstem.

In every case of unexplained stillbirth, according to this protocol, transverse serial histological sections are obtained from the midbrain, pons, medulla oblongata and the rostral part of the spinal cord, and treated with specific histochemical techniques for the nervous system staining (as Kluver/Barrera, Nissle, Bielchowsky). The microscopic examination allows to point out the morphology of the main nuclei and structures of the brainstem along their full length. They essentially are: the red nucleus and substantia nigra in the midbrain; the locus coeruleus and Kolliker-Fuse nucleus in the rostral pons; the retrotrapezoid nucleus, superior olivary complex and parafacial nucleus in the caudal pons; the hypoglossus, dorsal motor vagus, tractus solitarius, ambiguus, pre-Bötzinger, inferior olivary, raphe and arcuate nuclei in the medulla oblongata and the intermediolateral nucleus in the rostral spinal cord. Figure 1 shows a schematic representations of the localization of these nuclei in the sections obtained from different levels of the brainstem. In addition to the morphological examination, the application of immunohistochemical techniques to highlight the expression of specific biological markers (as catecholamines, serotonin, somatostatin, nicotinic receptors, orexin, etc.) can offer useful information on the phyisiology of the above mentioned structures $(5,6)$.

Thanks to this methodology of study, the "Lino Rossi" Research Center has identified subtle developmental anomalies, prevalently hypoplasia, of many nuclei of vital importance in sudden unexplained deaths, not observable in age-matched victims who died of known cause. These alterations, as reported in numerous publications (available at: http://users.unimi.it/centrolinorossi/en/publications.html), have offered a plausible explanation of the pathogenetic mechanism leading to sudden death in utero. Very interesting is the observation, in the great part of unexplained stillbirths, of hypoplasia of the parafacial nucleus, a roundish cluster of large polygonal neurons in the caudal pons, so called for its position adjacent to the 


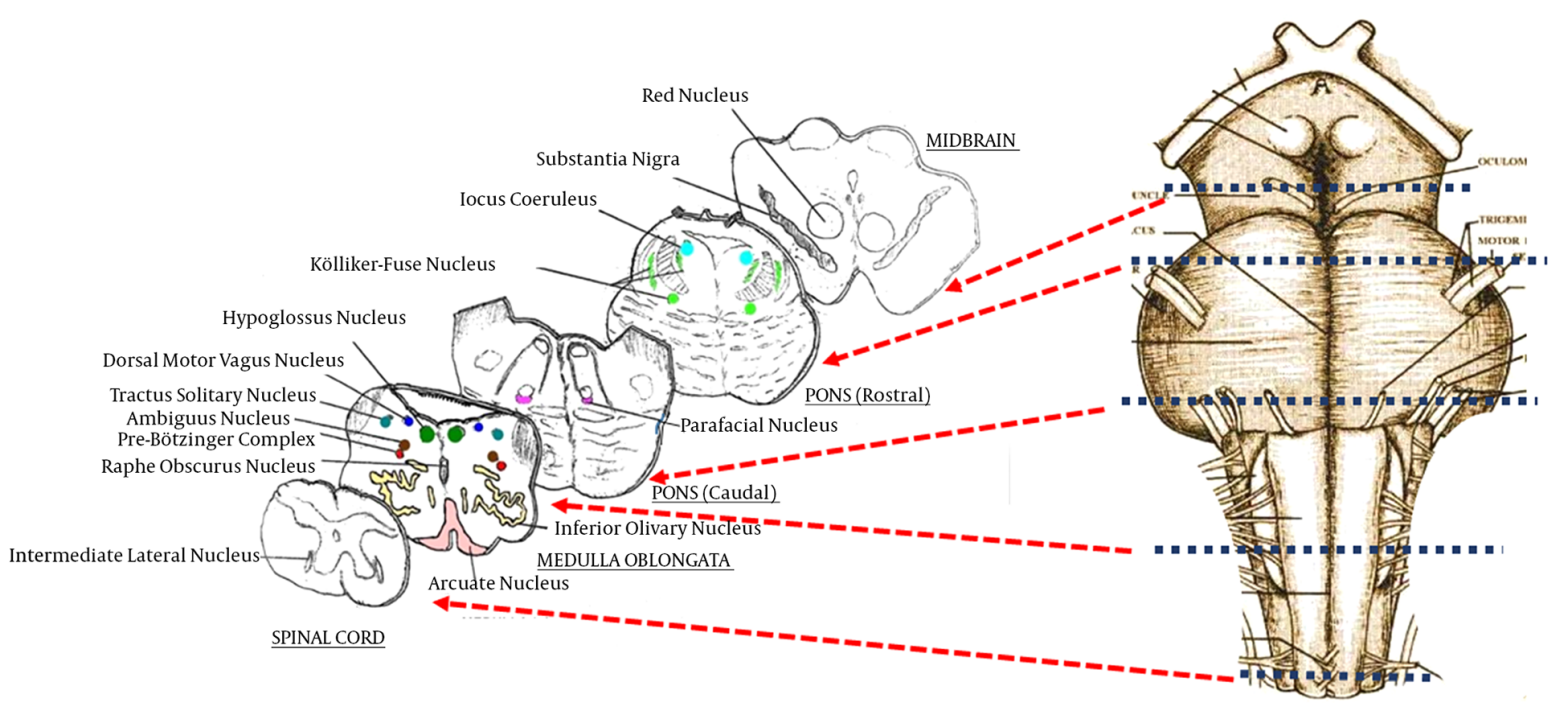

Figure 1. Schematic representation of the histological sections obtained at different levels of the brainstem, indicating the localization of the main nuclei and structures to be examined.

facial nucleus. The parafacial nucleus is a component of the "respiratory network", to which also belong the Kölliker-Fuse nucleus, the pre-Bötzinger nucleus and the intermediolateral nucleus. These centers, connected one with each other, are able to regulate the respiratory activity before and after birth, through excitatory and/or inhibitory connections in relation to the need. In particular, the parafacial nucleus acts as master generator of the respiratory rhythm in mammals. It starts working only from birth, giving rise to the first inspiratory act and all subsequent ones through the activation of the pre-Botzinger nucleus (7)

The episodic respiratory activity which occurs in fetal life, essentially aimed to promote the lung development, is instead generated by another nucleus of the respiratory network, i.e. the intermediolateral nucleus, under control of the Kolliker-Fuse nucleus. It is spontaneous to wonder how the hypodevelopment of the parafacial nucleus can be a so frequent finding in unexplained stillbirths, when breathing is not a vital function.

An hypothesis, could be that, in the last weeks of pregnancy, advancing towards the birth, a general check of all the brainstem nuclei that coordinate the essential functions for the extra-uterine life, and in particular the respiratory activity, occurs. Unexpected fetal death could therefore be ascribed to a selective process of self-suppression in presence of developmental alterations of the respiratory network components, particularly of the parafacial nucleus, to avoid the most devastating event of a sudden infant death after birth.

Since the hypoplasia of the parafacial nucleus has never been found in our large series of SIDS, we suppose that the normal development of this nucleus is essential for extra-uterine life, and that its hypodevelopment can be considered a specific marker of unexplained stillbirths, or at least of a great subset of unexplained stillbirths.

It is important also to understand what risk factors are associated with stillbirth.

It is well known that the fetal brain is particularly vulnerable to injuries in critical phases of its development. Then we believe that the parafacial nucleus is more susceptible to environmental factor exposure in utero than other neuronal structures. Maternal smoking is worldwide recognized as the main potential risk factor for intrauterine sudden death $(5,8)$. Also maternal alcohol and drug abuse and air pollution have been implicated in defective brainstem development in stillbirth. A new factor, recently identified, is the place where the mother lives. Rural residence has been in fact reported to contribute to stillbirth, due to the use of pesticides in that area (9-11).

In conclusion, a systematic evaluation of the brainstem, possibly performed by experienced, reliable pathologists, associated to the analysis of all the potential risk factors, is extremely important to highlight any possible pathogenic mechanism in unexplained stillbirth and to establish appropriate preventive programs. 


\section{References}

1. MacDorman MF, Gregory EC. Fetal and perinatal mortality: United States, 2013. National vital statistics reports. Hyattsville: National Center for Health Statistics; 2015.

2. Gardosi J, Kady SM, McGeown P, Francis A, Tonks A. Classification of stillbirth by relevant condition at death (ReCoDe): population based cohort study. BMJ. 2005;331(7525):1113-7. doi: 10.1136/bmj.38629.587639.7C. [PubMed: 16236774].

3. Constitution of the Italian Republic Law $n^{\circ} 31$. Regulations for diagnostic post-mortem investigation in victims of sudden infant death syndrome (SIDS) and unexpected fetal death Official Gazette of the Italian Republic, General Series; 2006. Available from: http://users. unimi.it/centrolinorossi/files/gazz_ufficiale.pdf.

4. Roncati L, Pusiol T, Piscioli F, Barbolini G, Maiorana A, Lavezzi A. The First 5-Year-Long Survey on Intrauterine Unexplained Sudden Deaths from the Northeast Italy. Fetal Pediatr Pathol. 2016;35(5):315-26. doi: 10.1080/15513815.2016.1185751. [PubMed: 27308949].

5. Lavezzi AM, Cappiello A, Pusiol T, Corna MF, Termopoli V, Matturri L. Pesticide exposure during pregnancy, like nicotine, affects the brainstem alpha7 nicotinic acetylcholine receptor expression, increasing the risk of sudden unexplained perinatal death. $J \mathrm{Neu}$ rol Sci. 2015;348(1-2):94-100. doi: 10.1016/j.jns.2014.11.014. [PubMed: 25433450].
6. Lavezzi AM, Ferrero S, Roncati L, Matturri L, Pusiol T. Impaired orexin receptor expression in the Kolliker-Fuse nucleus in sudden infant death syndrome: possible involvement of this nucleus in arousal pathophysiology. Neurol Res. 2016;38(8):706-16. doi: 10.1080/01616412.2016.1201632. [PubMed: 27353953].

7. Lavezzi AM, Ferrero S, Matturri L, Roncati L, Pusiol T. Developmental neuropathology of brainstem respiratory centers in unexplained stillbirth: What's the meaning? Int J Dev Neurosci. 2016;53:99-106. doi: 10.1016/j.ijdevneu.2016.06.007. [PubMed: 27477774].

8. Lavezzi AM, Ottaviani G, Matturri L. Adverse effects of prenatal tobacco smoke exposure on biological parameters of the developing brainstem. Neurobiol Dis. 2005;20(2):601-7. doi: 10.1016/j.nbd.2005.04.015. [PubMed:15925516].

9. Roncati L, Termopoli V, Pusiol T. Negative Role of the Environmental Endocrine Disruptors in the Human Neurodevelopment. Front Neurol. 2016;7:143. doi: 10.3389/fneur.2016.00143. [PubMed: 27625632].

10. Roncati L, Pusiol T, Piscioli F, Lavezzi AM. Neurodevelopmental disorders and pesticide exposure: the northeastern Italian experience. Arch Toxicol. 2017;91(2):603-4. doi: 10.1007/s00204-016-1920-7. [PubMed: 28032145].

11. Roncati L, Piscioli F, Pusiol T. The endocrine disruptors among the environmental risk factors for stillbirth. Sci Total Environ. 2016;563564:1086-7. doi: 10.1016/j.scitotenv.2016.04.214. [PubMed: 27216966]. 